\title{
Control Based On the Temperature and Moisture, Using the Fuzzy Logic.
}

\author{
A. HILALI*, H. ALAMI** and A. RAHALI*** \\ * Faculty of Science, Department of physics, Laboratory of Electronics, Automatics and Biotechnology, PO Box \\ 12101, Zitoune, Meknes, Morocco. \\ **Faculty of Science, Department of physics, Laboratory of Electronics, Automatics and Biotechnology, PO \\ Box 12101, Zitoune, Meknes, Morocco. \\ ***Faculty of Science, Department of physics, Laboratory of Electronics, Automatics and Biotechnology, PO \\ Box 12101, Zitoune, Meknes, Morocco.
}

\begin{abstract}
This paper explains the design and implementation of an electronic system based on a for remote control of several experimental greenhouses. This system enables its user to consult the climatic parameters and to order the greenhouses sub-systems equipment's by SMS. The climate Sensors are packaged using the electronic circuits, and the whole is being interfaced with maps of acquisitions (Arduino) via a radio frequency connection. These sensors provide information used for the control of ventilation, heating and water pumping by SMS. The acquisitions boards contain fuzzy controllers who manage the climate for local agricultural greenhouses. The procedure used in our system offers the operator an optimal control and monitoring without traveling to the place where the greenhouses are located, using his mobile phone, and being able to view at any moment the state of the greenhouse climate via the send and receive SMS function.
\end{abstract}

Keywords: Arduino, climate control, fuzzy logic, Greenhouse network, GSM

\section{INTRODUCTION:}

The greenhouse is a simple enceinte limited by a transparent wall that isolates the culture from the adverse effects of the local climate. To achieve the predefined objectives of the greenhouse, first, it is compulsory to choose the type of material that is used in the coating and the insulation of the greenhouse against the undesirable external effects. The choice of coating materials for the greenhouse is the most important part of the whole process. It is the main element responsible for losses of energy within the greenhouse [1], [2]. Hence, Network materials for recovery are available [3], [4].

The use of greenhouses systems, managing climate parameters, is becoming more and more critical and essential. As it allows to maintain the neighborhoods in a predefined climate. These are adjusted to achieve two main objectives: to maximize the production within greenhouses [5], [6] and to protect the culture under shelter against the incidental diseases during its growth process [7], [8].

The production within a greenhouse helps to alleviate the problems encountered when a culture is being raised in an open air, where the needs of crops are limited with the local climate and also the external climatic conditions are preventing production under greenhouses. The greenhouse allows you to easily manage the climatic factors which are not the case for a culture in the open air. Among the major benefits of a production within a greenhouse, we state: maximizing production thanks to the possibility of controlling the climatic conditions of the culture in fostering the production at all seasons of the year, the increase in the yield and quality of the crop, the precocity of the production and the reduction of the consumption of fungicides and insecticides.

Since the beginning of the under-shelter crops, the climate management has become the key link in this type of production. The control of climate parameters, taking more and more into account the management of the energy and resources, is therefore part of the major issues with a view to reducing energy consumption and the impact on the environment of these areas of culture.

Mastering both of the climate and irrigation parameters, is of a vital importance for most of the plants grown under greenhouses. Therefore, the use of specific regulators is needed, given the number of parameters to control. These regulators allow equipment control within the greenhouse in order to respect the climate instructions. A couple of sensors can be installed in the inside as in the outside of greenhouses. Thanks to accuracy sensors and effective programs, it is easy to optimize the management of the greenhouse related to certain parameters such as temperature, 
relative humidity, light, and the content of carbon dioxide. In reality, each of these parameters generates a combination of effects that can be favorable or not in the functioning of the emissions according to the local conditions.

After the practical implementation of network strategies of commands such as the TOR (All or Nothing) control, the command by cycle of hysteresis, the PID and PI-intelligent [9], the logic Gaussian quadratic [10], and the fuzzy controller for the temperature and humidity inside the greenhouse [11], it has been thought to put into practice an embedded system, while introducing the maximum of its algorithms of commands to simplify to users the use of its system and promote the good climate of greenhouses.

A well mastered monitoring of the climate allows therefore to manage these settings and to improve the physiological functioning of plants [12]. In this perspective, we have developed a device allowing the regulation of the climatic conditions of a network of greenhouses. The latter, ensures an automation of all of the equipment for each sub-system greenhouse. It also incorporates many of the settings in intelligent management in order to ensure the best agricultural performance while providing a better quality of use to the farmer.

\section{THE STRUCTURE OF THE DEVELOPED SYSTEM:}

The following figure represents the structure of the developed system:
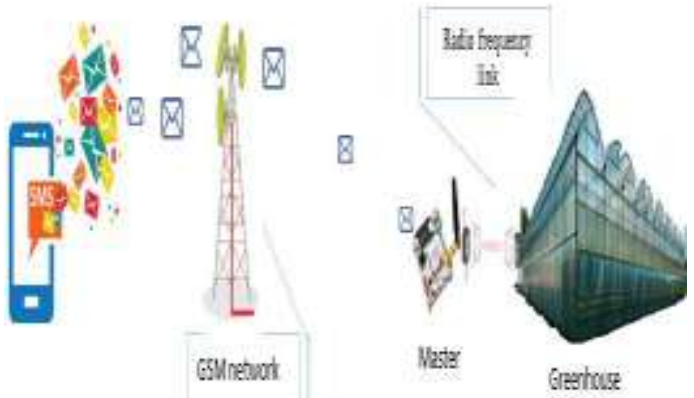

Fig. 1: System Functional Schematic

Figure 1, shows the structure of the greenhouse system controlled by GSM. The system allows the acquisition of different climate parameters (internal and external temperature, internal and external humidity, soil moisture), and command the actuators. A GSM module with integrated modem allows the system to be controlled on the whole extent of coverage of the GSM network. An authentication based on the content of the commands is systematically performed to ensure security on one hand and to allow on the other hand the use of any mobile phone. The remote functions are: Opening / closing of roofs, triggering / switching of the irrigation in a modulating way by solenoid valve, on / off of ventilation and heating. The SMS issued by the system to the user are intended to notify any detection and confirm that the commands are being taken into account.

\section{THE CONSTITUTING OF THE SYSTEM}

The following figure shows the local structure of the system

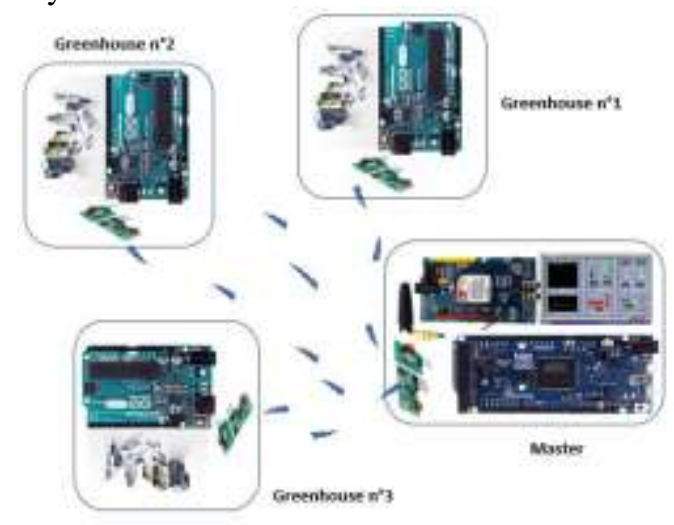

Fig. 2: the structure of system

The main system is designed around an acquisition card connected mainly with a GSM modem, a micro SD memory card to save the history of SMS and climatic parameters of each system, a graphical interface and the other three cards of acquisitions of three systems greenhouses. The communication between the different maps is therefore ensured by modules transmitters/receivers radiofrequency type amplitude modulation to carrier frequency of $433 \mathrm{Mhz}$.

Figure 3 shows the main interface dynamic graphic intended to notify any detection or confirm the taking into account of the commands. And also allows you to display the evolution in real time of all the parameters of the Sub-local systems. This interface is composed of three sub-interfaces each of them is designed for a well defined greenhouse system. These can be set and configured, with the possibility of changing instructions or turning on and off certain features of such a system, and eventually the possibility of managing few alarms.

Some plants require some very specific climatic conditions, which must be favorable to the culture in question, to have a good growth and performance. The management of alarms is used to take into account this kind of requirements. In the Settings window, you have the possibility to define limit values of each parameter climate. As soon as there is an over run the system intervenes and turns on a sound alarm, then sends an SMS to the owner 
and subsequently issues a phone call after which it acts on some power equipment in order to solve the problem. The account of each outbreak will be saved in the Alarm window. Figure 3 shows the possibilities offered by the local management of the greenhouse number one, after having clicked on the button connected on the reserved area.

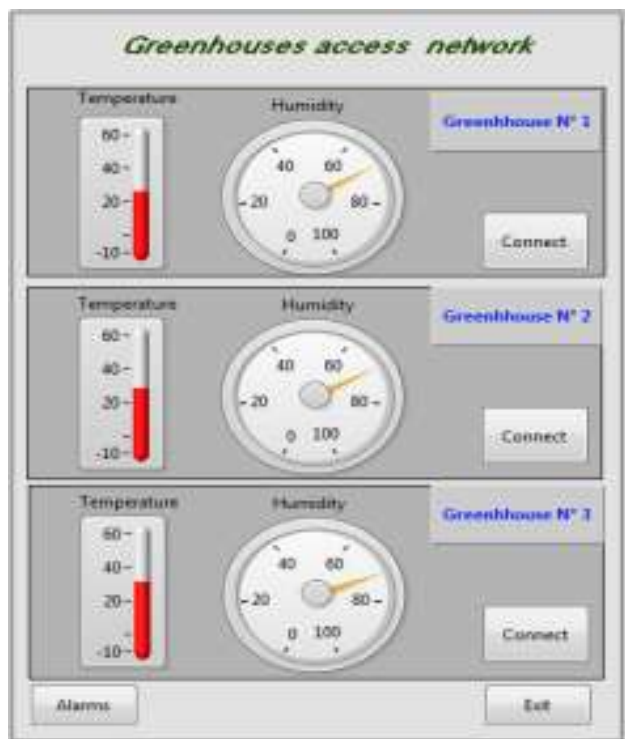

Fig.3: Interface of supervision of the master

The figure below shows the interface of the Supervision developed under LabVIEW, the user can locally check the climate of the PLANTS in a very friendly manner where the details are represented in a graphical interface dynamic, as it can display the history of the messages sent and possibly the previous measures of the sensors using the button ' History " indicated on the figure, in addition to the possibility of reconfiguring each greenhouse system.

The essential features of the pilotage system carried out allow you to perform the following functions: storage of Data, Supervision in real time of the set of parameters and Remote control via GSM.

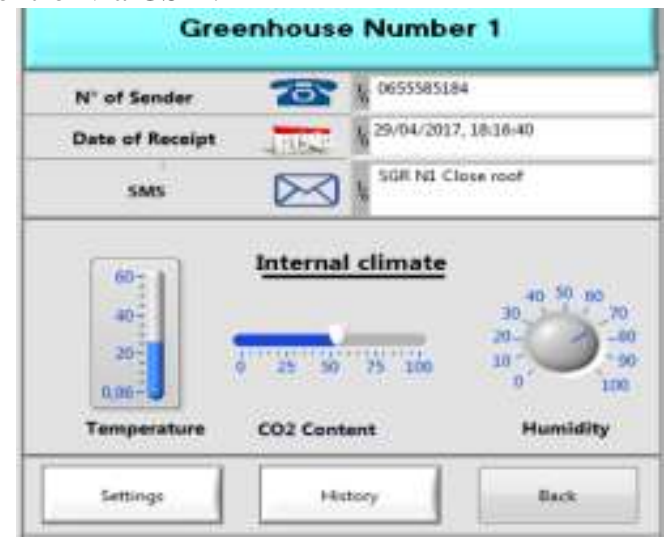

Fig.4: Graphical interface of the greenhouse 1
The data displayed on each interface is obtained via a Radio Frequency link and thanks to an Arduino board installed on each greenhouse, this card ensures the acquisition of the data of each agricultural greenhouse. The network of sensors (temperature, humidity, light and carbon dioxide) and actuators (Heating, the fan and valves of irrigations) are related to the acquisition card, this card communicates with the master via a transmitter- receiver radio frequency with a scope that is going up to two kilometers.

Each command card (Arduino) is the main axon of each sub-system. It contains the program of fuzzy controller for the regulation of temperature and humidity, acquires the information from the various sensors, communicates with the powers organs through the below-actuators, backs- up all its measures periodically on a support SD memory and sends its measures to the main system.

A schematic diagram of the fuzzy controller proposed is shown in Figure 5. We would like to order the duration of irrigation(Dirr)by implementing the fuzzy controller under an Arduino board. This controller has two inputs, the interior temperature (TIn)and the moisture of the soil (hs). The system has an output signal that controls the duration of irrigation(Dirr).

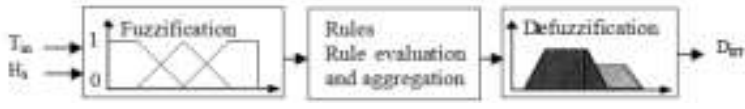

Fig. 5 : Diagram of the fuzzy controller proposed

\section{THE STRUCTURE OF THE FUZZY CONTROLLER}

The fuzzy controller developed is composed of two modules:

\section{Fuzzification of the Fuzzy Controller}

We established the quantifiers fuzzy and functions of belonging to the entries in the system. For the fuzzification of the indoor temperature(Tin), we choose five intervals fuzzy and of the functions of memberships in trapezoidal types and triangular. The range of the universe of the speech of the variable of language "TIN" is $0^{\circ} \mathrm{C}$ to $50^{\circ} \mathrm{C}$. The predicates "Very_Cold, Cold, Normal, Hot and Very_Hot" are linguistic values of the variable "Tin." For each of these predicates, it combines a function of belonging $\mu$ predicate(x). Figure 6 illustrates the distribution blurred in the input variable such as the interior temperature(Tin). 


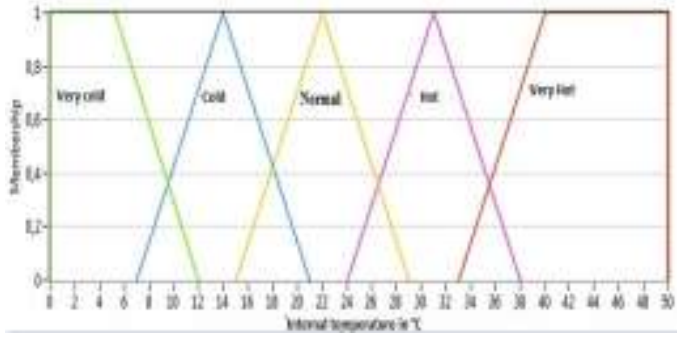

Fig. 6 : fuzzy quantification of the input variable inside temperature (TIn)

We choose three intervals fuzzy and of the functions of memberships in trapezoidal types and triangular for the fuzzification soil moisture in defining the "dry" as corresponding to a humidity less than $25 \%$, the predicate "wet" as a humidity between $40 \%$ and $50 \%$ and the predicate "very wet" as being a higher moisture to $70 \%$. Figure 7 illustrates the distribution blurred in the input variable soil moisture (hs).

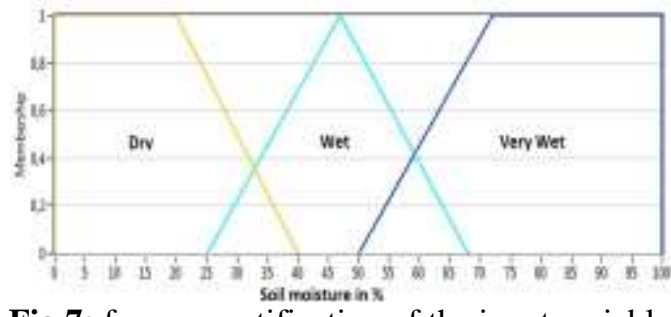

Fig.7: fuzzy quantification of the input variable soil moisture (hs) in percent

Subsequently, we established the quantifiers fuzzy and functions of belonging to the output of the system. We chose three intervals fuzzy and functions of membership of trapezoidal type by defining the predicate "short" as corresponding to a duration of irrigation less than 10 minutes, the predicate "average" as being a duration of irrigation between 20 and 32 minutes and the predicate "long" as being a duration of irrigation greater than 42 minutes. Figure 8, illustrates the distribution blurred the output variable duration of irrigation(Dirr).

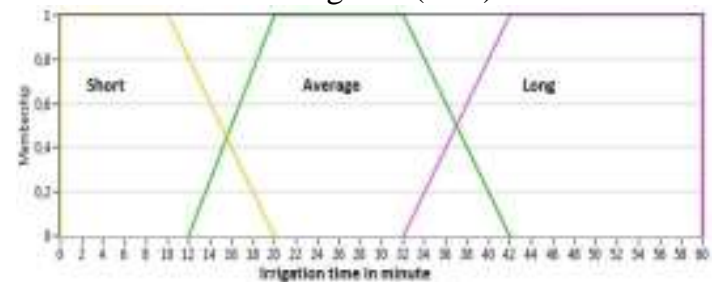

Fig. 8: fuzzy quantification of the output variable duration of irrigation (Dirr)

\section{The Basis of the rules of the Fuzzy Controller}

The experience obtained of the drip irrigation system used under greenhouses has helped define the fifteen rules of the system shown in the following table 1 :
Table1: Rules of the fuzzy controller proposed

\begin{tabular}{|l|l|l|l|}
\hline Rule & IF Tin & And Hs & $\begin{array}{l}\text { Then } \\
\text { Dirr }\end{array}$ \\
\hline 1 & Very cold & Dry & Long \\
\hline 2 & Very cold & Wet & Short \\
\hline 3 & Very cold & Very wet & Short \\
\hline 4 & Cold & Dry & Long \\
\hline 5 & Cold & Wet & Average \\
\hline 6 & Cold & Very wet & Short \\
\hline 7 & Normal & Dry & Long \\
\hline 8 & Normal & Wet & Average \\
\hline 9 & Normal & Very wet & Short \\
\hline 10 & Hot & Dry & Long \\
\hline 11 & Hot & Wet & average \\
\hline 12 & Hot & Very wet & Short \\
\hline 13 & Very hot & Dry & Long \\
\hline 14 & Very hot & Wet & Average \\
\hline 15 & Very hot & Very wet & short \\
\hline
\end{tabular}

The method of inference chosen is the MAMDANI one. Therefore, the operator AND is carried out by the calculation of the minimum, while the OR operator is realized by the calculation of the maximum [13].

\section{CONCLUSION}

The objective of this Article is to offer to farmers a control system for greenhouses that will be automatically performing remotely via GSM, which can meet their needs. The system is controlled by a master that is currently a privileged tool for data acquisition. This system has multiple benefits, namely the power of execution, the availability of Interfaces of control and monitoring, and also the level of data storage capacity. The developed program will allow to exploit greenhouses during the whole year. Different experimental methods have been used in our laboratory to regulate the climate parameters under greenhouse: [14],[15],[16],[17].However, such methods are sometimes difficult to implement, the fuzzy logic seems to be a good compromise of practical choice to perform a multivariate command while introducing a supervision, which takes into account both the state of the process and its behavior and of the value of the perturbations of conditions of crops.

\section{REFERENCES}

[1]. M.A. Olmstead and J.M. Tarara. Physical principles of row covers and grow tubes with application to small fruit crops. Small Fruits Review 1(3), 29-46 (2001).

[2]. B. Cemek and Y. Demir. Testing of the condensation characteristics and light transmissions of different plastic film 
covering materials. Polymer testing 24(3), 284-289 (2005).

[3]. A. Baille. Serres plastiques, climat et production. Pépiniéristes Horticulteurs Maraîchers (PHM) Revue Horticole 357, 15-19 (1995).

[4]. G.A. Giacomelli and W.J. Roberts. Greenhouse covering systems. HortTechnology 3(1), 50-58 (1993).

[5]. M. Trigui, S. Barrington, and L. Gauthier. Structures and environment : A strategy for greenhouse climate control, part i : Model development. Journal of agricultural engineering research 78(4), 407-413(2001).

[6]. F. Rodríguez, M. Berenguel, and $M$. Arahal. A hierarchical control system for maximizing profit in greenhouse crop production. Proceeding in European Control Conference ECC03, Cambridge, UK (2003).

[7]. T. Hans-Juergen and L. Doris. Greenhouse climate control : an approach for integrated pest management. Computers and Electronics in Agriculture 40(1-3), 141-152 (2003).

[8]. R. J. William. Managing diseases in greenhouse crops. APS Press St. Paul. MN. US(1992)

[9]. Y. El Afou1, L. Belkoura, M. Outanoute, M. Guerbaoui, A. Rahali, A. Ed-Dahhak, A. Lachhab, C. Join, B. Bouchikhi. Feedback Techniques Using PID and PIIntelligent For Greenhouse Temperature Control. International Journal of Advanced Research in Electrical,Electronics and Instrumentation Engineering Vol. 3, Issue 6, June (2014).

[10]. M. Outanoute, A. Lachhab, A. Ed-dahhak, M. Guerbaoui, A. Selmani, B. Bouchikhi. Synthesis of an Optimal Dynamic Regulator Based on Linear Quadratic Gaussian (LQG) for the Control of the Relative Humidity under Experimental Greenhouse. International journal of Electrical and Computer Engineering (IJECE) Vol. 6 , No 5 (2015).

[11]. A.Ed-dahhak, M. Guerbaoui, Y. ElAfou, M. Outanoute, A. Lachhab,L. Belkoura and B. Bouchikhi. Implementation Of Fuzzy Controller To Reduce Water Irrigation In Greenhouse Using Labview. International Journal of Engineering and Advanced Technology Studies Vol.1 No. 2, pp. 12-22, September 2013.

[12]. Mistriotis A., Arcidianco C, Picuno P, Bot G.P.A., Sacarscia-Mugnozza G. Computational analysis of ventilationin greenhouses at zero-and low- windspeeds. Agricultural and forest Meteorology, 88, 121-135, 1997.

[13]. Mamdani E., Assilian S., 1975. An experiment in linguistic synthesis with a fuzzy logic controller, International Journal of Man-Machine studies, 7(1), 113.

[14]. Lachhab A., Ezzine L., Ed-Dahhak A., Didi S., Salinas Vazquez J. R., Garcia Lagos F., Atencia M., Joya Caparros G. et Bouchikhi B., 2008. Identification of greenhouse dynamic temperature model using statistical modelling AMSE Journal Modelling D, 29 (2), 38-48.

[15]. Ed-Dahhak A. Development of a system for monitoring the climate and managing the drip fertilizing irrigation in greenhouse by using LabVIEW software. National $\mathrm{PhD}$, Faculty of Sciences, Meknes, Moulay Ismail University, Morocco. , 2009.

[16]. Ezzine L., Guerbaoui M., El Afou Y., EdDahhak A., Lachhab A., Belkoura L., Bouchikhi B., Implementation of identification algorithm using Recurrent RBF Neural Networks for the inside temperature in a greenhouse, International Mediterranean \& Latin American Modelling Multi conference I3M Fes, Morocco, 13-15 October. 2010.

[17]. Guerbaoui M., El Afou Y., Ed-Dahhak A., Lachhab A., Bouchikhi B., 2013. PCBased Monitoring For Drip Irrigation System, International Journal of Engineering Science and Technology, 5(1), 221-225.

[18]. Hayat Khiyal M. S., Khan A. and E. Shehzadi. SMS Based Wireless Home Appliance Control System (HACS) for Automating Appliances and Security. Issues in Informing Science and Information Technology, 6, 887-8947, 2009.

[19]. Jiang P., Xia H., Zhiye He Z. and Wang Z. Design of awater Environment monitoring system based on wireless sensor networks. Journal of Sensors, 9, 6411-6434., 2009.

[20]. A Rahali, M Guerbaoui, A Ed-dahhak, Y El Afou, A Tannouche, A Lachhab, B Bouchikhi. Development of a data acquisition and greenhouse control system based on GSM. International Journal of Engineering, Science and Technology .Vol 3, No 8 (2011). 